Supporting Information for

Tuning of Network Dimensionality and Photoluminescent Properties in Homo- and Heteroleptic Lanthanide Coordination Polymers

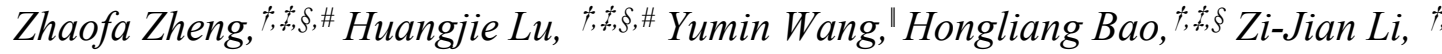

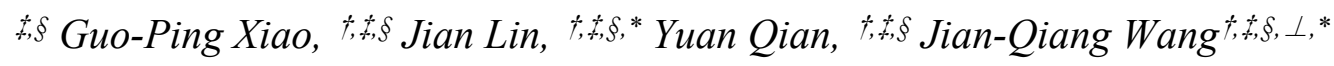

†Shanghai Institute of Applied Physics, Chinese Academy of Sciences, 2019 Jia Luo Road, Shanghai 201800, China.

†ey Laboratory of Interfacial Physics and Technology, Chinese Academy of Sciences, 2019 Jia Luo Road, Shanghai 201800, China.

§University of Chinese Academy of Sciences, No.19(A) Yuquan Road, Shijingshan District, Beijing, 100049, China.

"School for Radiological and Interdisciplinary Sciences (RAD-X) and Collaborative Innovation Center of Radiation Medicine of Jiangsu Higher Education Institutions, Soochow University, 199 Ren'ai Road, Suzhou 215123, China.

${ }^{\perp}$ Dalian National Laboratory for Clean Energy, Dalian 116023, China. 


\section{Table of Content}

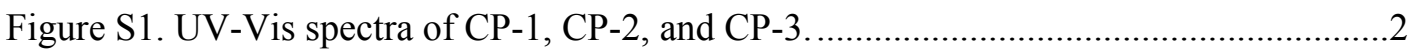

Figure S2. SEM images and EDS spectra of CP-1, CP-2, and CP-3 ...................................

Figure S3. The powder X-ray diffraction (PXRD) patterns of (a) CP-1, (b) CP-2, and (c)

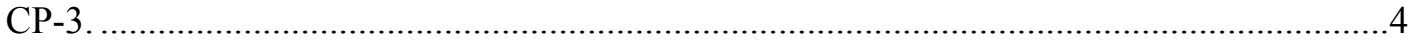

Figure S4. FTIR spectra of CP-1, CP-2, CP-3, and ligands. …............................................

Figure S5. The TGA plots of CP-1-Eu, CP-2-Eu, and CP-3-Eu. ........................................

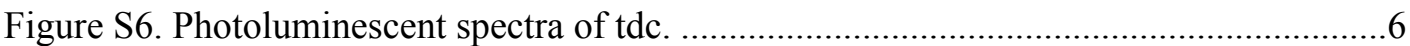

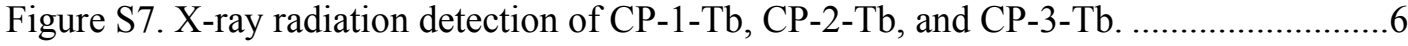

Figure S8. The EPR plots of CP-1-Tb, CP-2-Tb, and CP-3-Tb. ..........................................

Table S1. The comparisons of UV detection limits between $\mathrm{CP}-2-\mathrm{Tb}$ and other reported

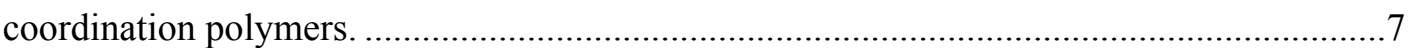




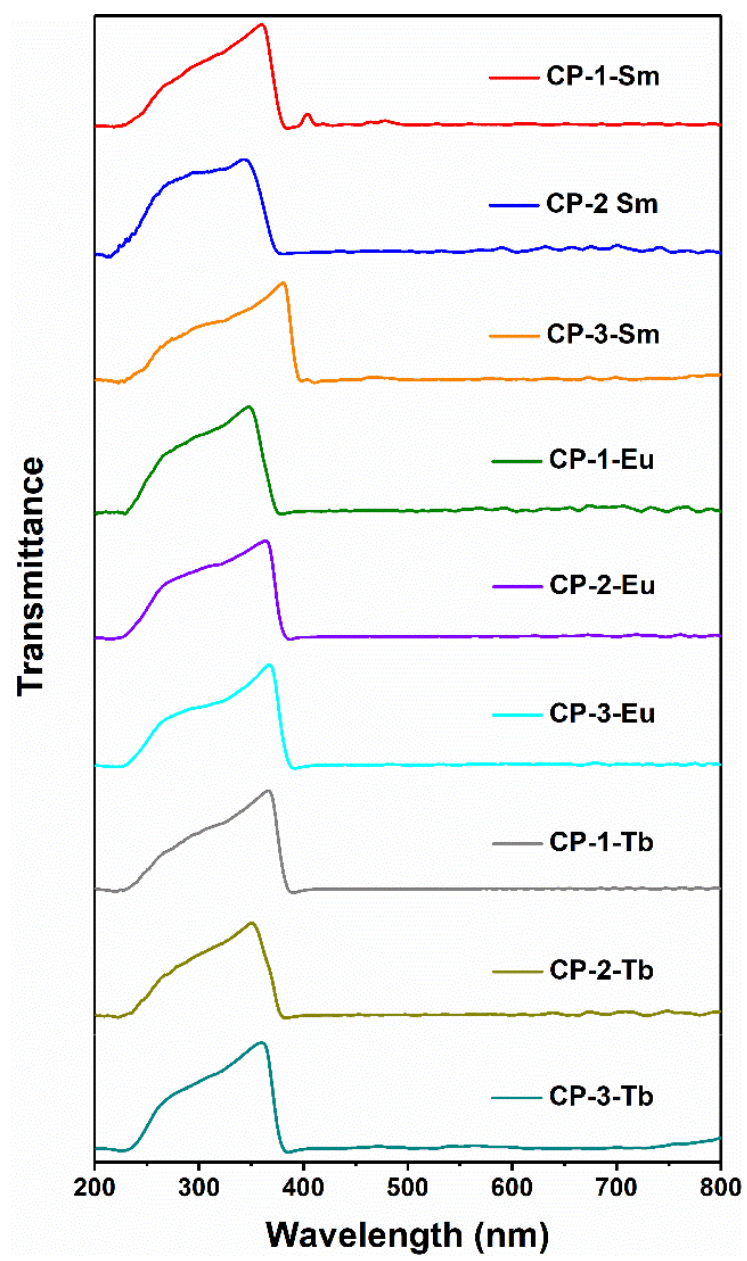

Figure S1. UV-Vis spectra of CP-1, CP-2, and CP-3.

2 

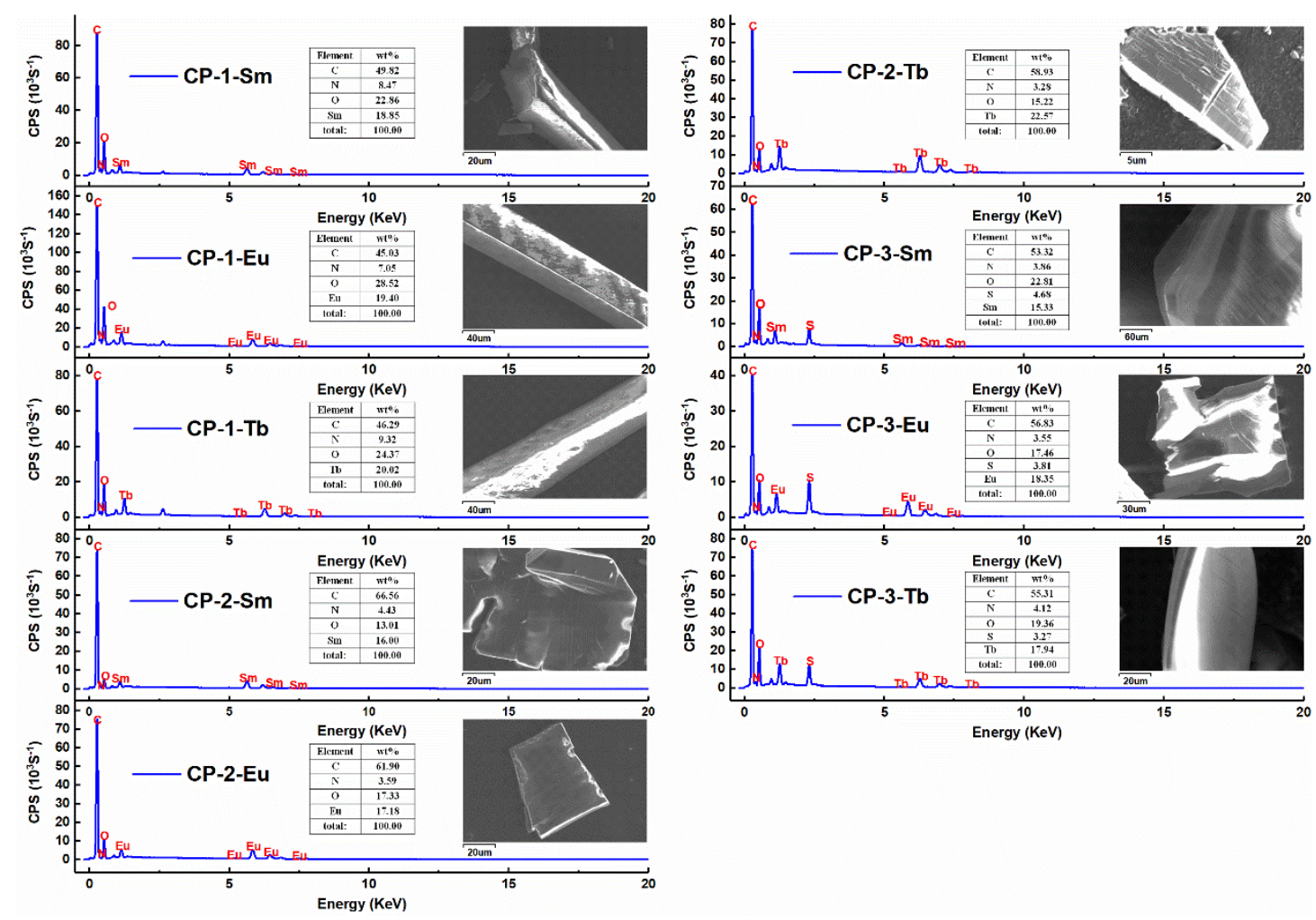

Figure S2. SEM images and EDS spectra of CP-1, CP-2, and CP-3. 



Figure S3. The powder X-ray diffraction (PXRD) patterns of (a) CP-1, (b) CP-2, and (c) CP-3. 

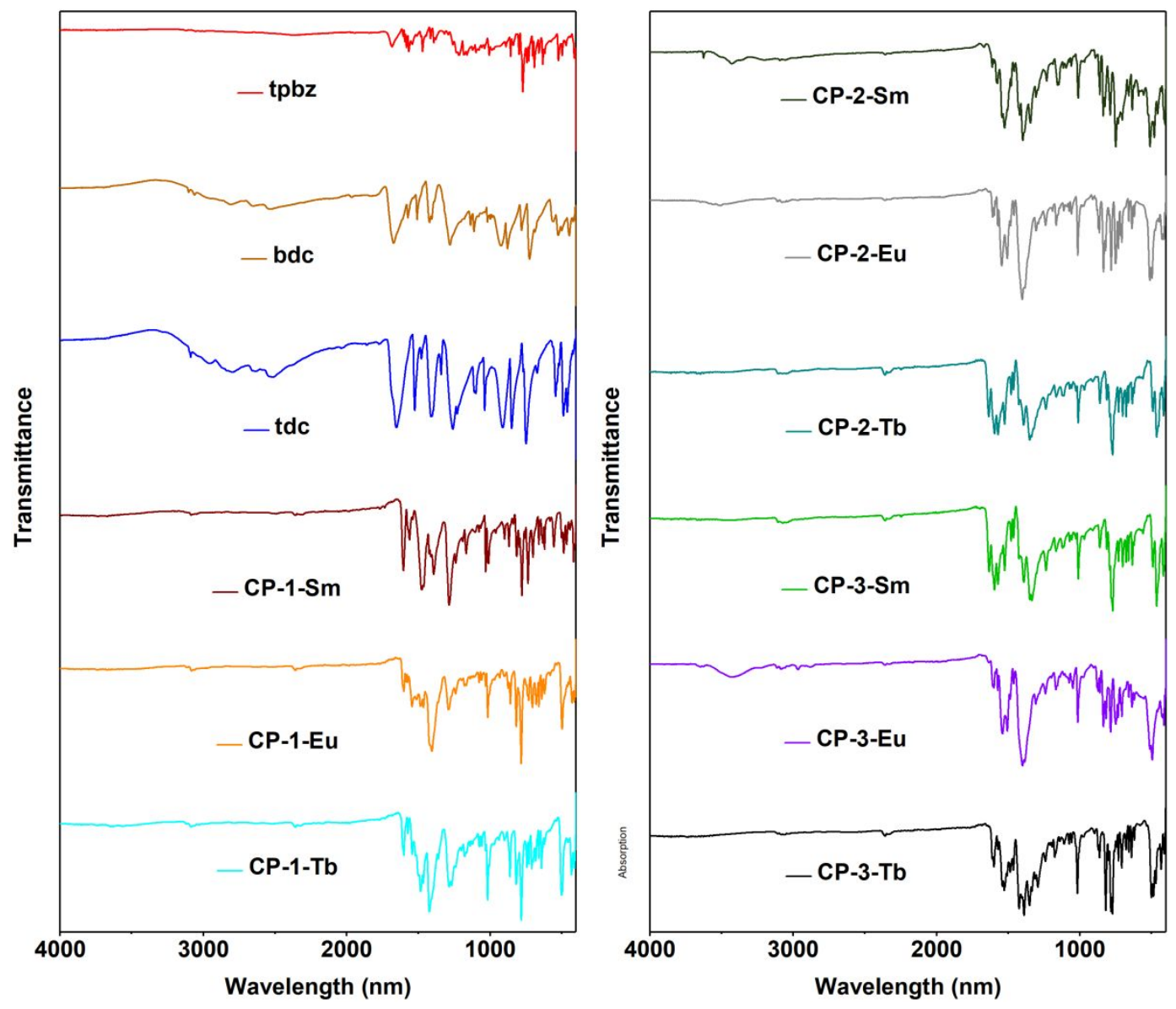

Figure S4. FTIR spectra of CP-1, CP-2, CP-3, and ligands.

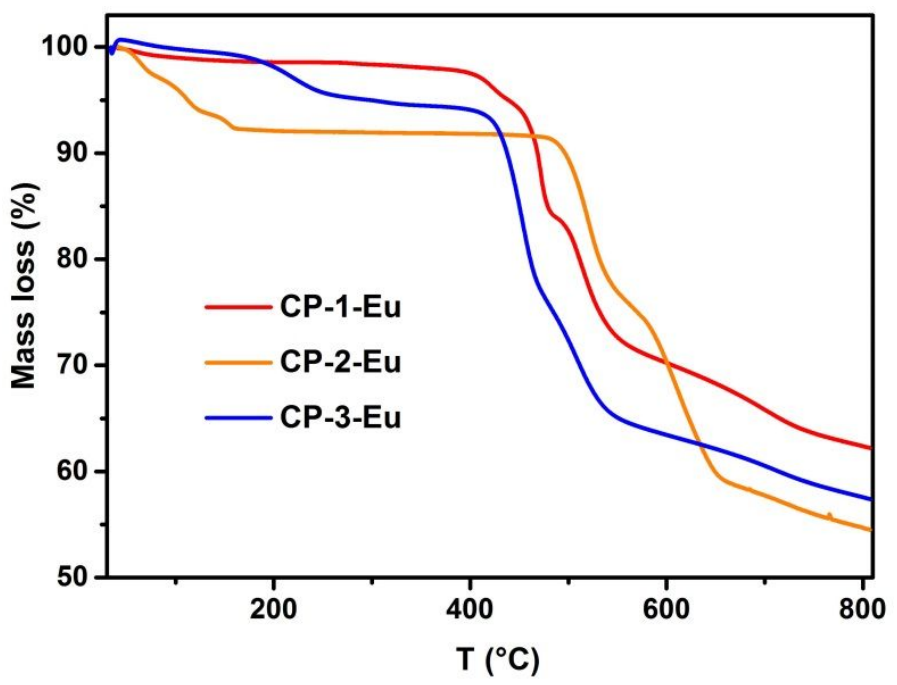

Figure S5. The TGA plots of CP-1-Eu, CP-2-Eu, and CP-3-Eu. 


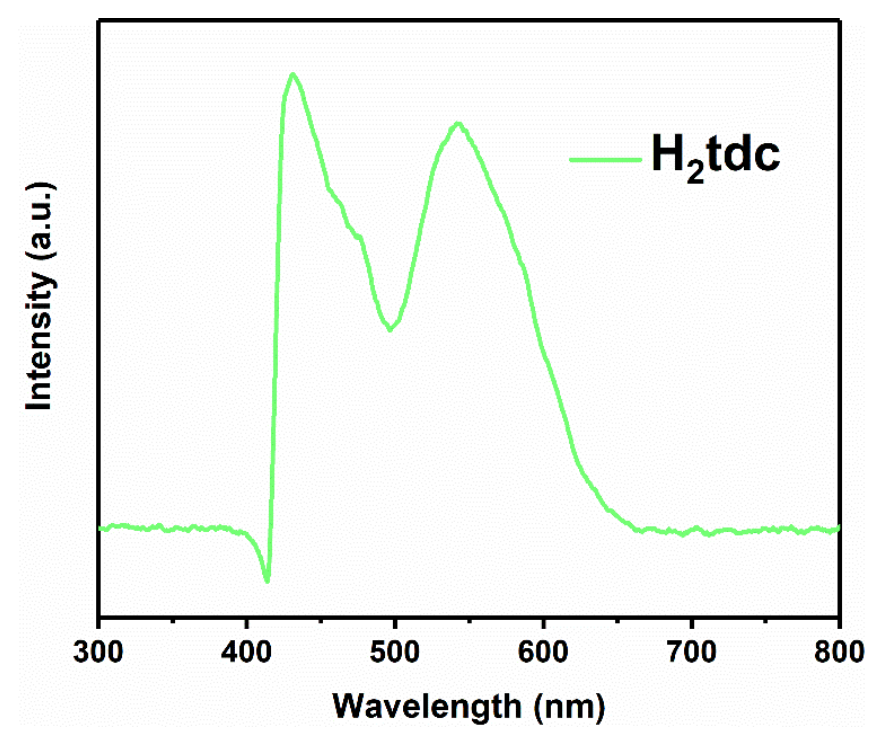

Figure S6. Photoluminescent spectrum of $\mathrm{H}_{2} \mathrm{tdc}$.
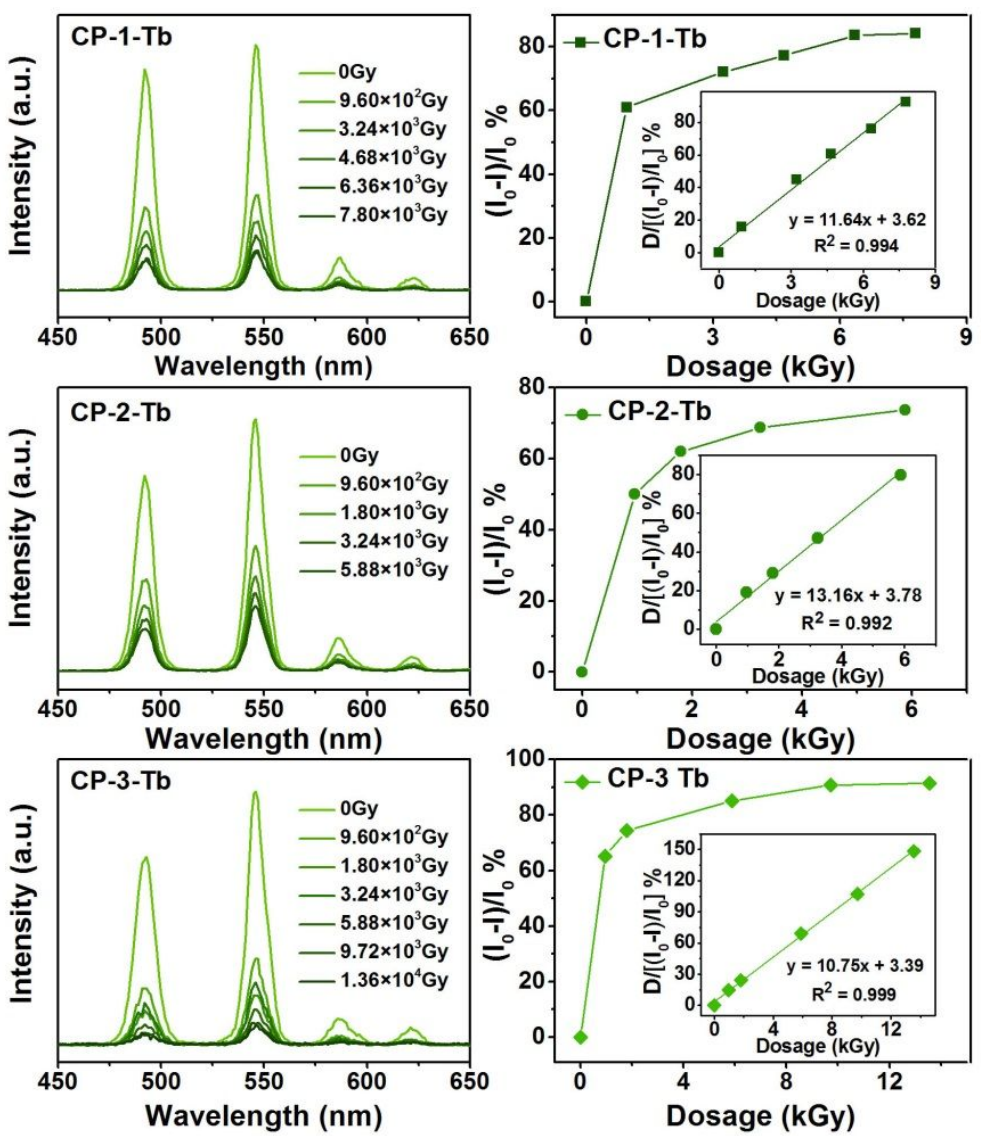

Figure S7. X-ray radiation detection of CP-1-Tb, CP-2-Tb, and CP-3-Tb. 


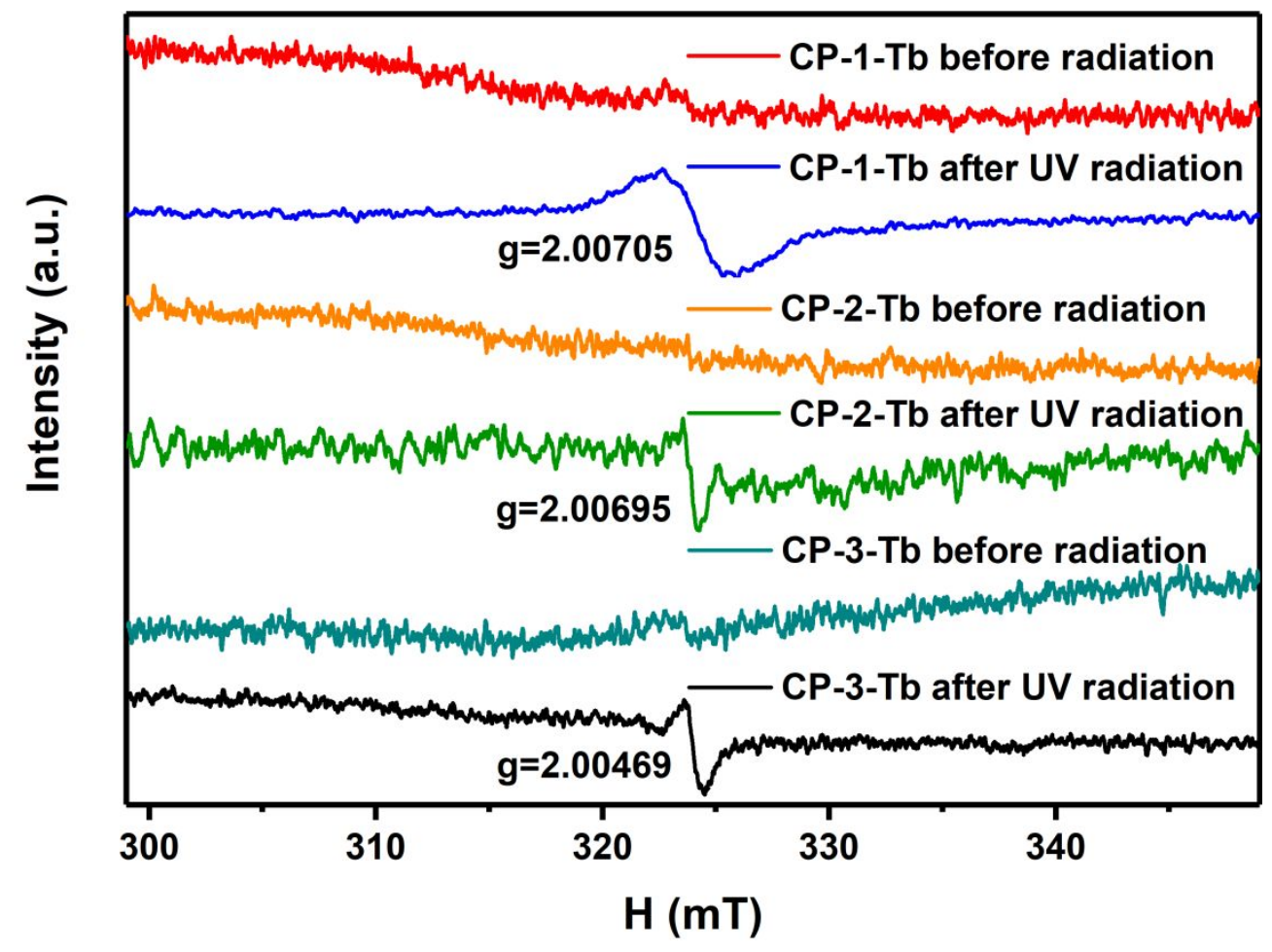

Figure S8. The EPR plots of CP-1-Tb, CP-2-Tb, and CP-3-Tb.

Table S1. The comparisons of UV detection limits between CP-2-Tb and other reported coordination polymers.

\begin{tabular}{lll}
\hline Coordination Polymers & Detection Limit & Reference \\
\hline$\left[\mathrm{UO}_{2}(\mathrm{~L})(\mathrm{DMF})\right]$ & $2.4 \times 10^{-7} \mathrm{~J}$ & 1 \\
{$\left[(\mathrm{TEA})_{2}\left(\mathrm{UO}_{2}\right)_{5}(\mathrm{PhPC})_{6}\right.$} & $3.9 \times 10^{-6} \mathrm{~J}$ & 2 \\
{$[\mathrm{Hphen}]_{2}\left[\left(\mathrm{UO}_{2}\right)_{2}(\mathrm{ox})_{3}\right]$} & $6.9 \times 10^{-9} \mathrm{~J}$ & 3 \\
$\mathbf{C P - 2}-\mathbf{T b}$ & $3.2 \times 10^{-7} \mathrm{~J}$ & This work \\
\hline
\end{tabular}




\section{References}

1. Liu, W.; Dai, X.; Xie, J.; Silver, M. A.; Zhang, D.; Wang, Y.; Cai, Y.; Diwu, J.; Wang, J.; Zhou, R.; Chai, Z.; Wang, S. Highly Sensitive Detection of UV Radiation Using a Uranium Coordination Polymer. ACS Appl. Mater. Interfaces 2018, 10, 4844-4850.

2. Chen Lei, C. L., Zhang Yugang, Xie Jian, Diwu Juan. Layered Uranyl Coordination Polymer with UV Detection Sensitivity, Stability, and Reusability. J Inorg. Mater. 2020, DOI: $10.15541 / \mathrm{jim} 20200139$.

3. Xie, J.; Wang, Y.; Zhang, D.; Liang, C.; Liu, W.; Chong, Y.; Yin, X.; Zhang, Y.; Gui, D.; Chen, L.; Tong, W.; Liu, Z.; Diwu, J.; Chai, Z.; Wang, S. Photo-exfoliation of a highly photoresponsive two-dimensional metal-organic framework. Chem. Commun. 2019, 55, 1171511718. 